\title{
Is more better than less? Caveats from bevacizumab and cetuximab combination in colorectal cancer
}

\author{
Camillo Porta
}

Published online: 4 March 2009

(c) Springer-Verlag 2009

In the past few years, impressing improvements have been made in the treatment of advanced colorectal cancer. Following decades of modest achievements, in which it was just a matter of dose and schedule for 5-FU and leucovorin - the only treatment then available-first, the development of irinotecan and oxaliplatin, and then the use of the two biologicals, bevacizumab and cetuximab, have dramatically improved the life expectancy of our colorectal cancer patients [1].

Therefore, a lot of expectations surrounded the first trial in which a combination of chemotherapy with both biologicals was tested. Such a combination was also supported by preclinical studies in animal models suggesting that combining a drug that targets vascular endothelial growth factor (VEGF) with a drug that targets epidermal growth factor receptor (EGFR) may be more effective than either drug alone; furthermore, single case reports suggested that such an increate activity could also be observed in the clinical setting [2].

The idea was to demostrate that we can obtain a better and longer disease control targeting two different, but nevertheless key, mechanisms responsible for tumor growth and spread, i.e., VEGF-driven angiogenesis, as well as the EGFR pathway.

This randomized clinical trial, testing chemotherapy combined with the VEGF-neutralizing monoclonal antibody Bevacizumab and the anti-EGFR monoclonal antibody cetuximab, and comparing this with chemotherapy and

C. Porta $(\square)$

Medical Oncology and Laboratory of Pre-Clinical Oncology and Developmental Therapeutics,

IRCCS San Matteo University Hospital Foundation, piazzale C. Golgi 19, 27100 Pavia, Italy

e-mail: c.porta@smatteo.pv.it bevacizumab alone, found that the addition of cetuximab actually shortened patients' recurrence-free and median survival, as reported in the February 5 issue of the New England Journal of Medicine [3].

Investigators from the Netherlands enrolled 755 patients with metastatic, inoperable colon or rectal cancer into this trial, continuing treatment until disease progression, death, or unacceptable toxicity.

Unfortunately, they found that "... the addition of Cetuximab significantly decreased the median progression-free survival ..." from 10.7 to 9.4 months; furthermore, median overall survival was 20.3 months for patients receiving chemotherapy and bevacizumab, versus 19.4 months for patients receiving chemotherapy, bevacizumab and cetuximab; finally, patients who received cetuximab reported less improvement in overall quality of life during treatment [3].

The investigators analyzed these results considering the status of $K R A S$, either wild type or mutated. A number of studies have indeed shown that only patients with normal $K R A S$ benefit from drugs targeting EGFR [4]. Patients with mutant KRAS who were given cetuximab had a significantly shorter progression-free survival than patients treated with chemotherapy and bevacizumab, while, for patients with normal KRAS, cetuximab had no effect on progression-free survival [3].

The cause of these unexpected negative results are presently not clear, although a negative interaction between the two biologicals has been postulated as a possible mechanism responsible for such unexpectedly unsatisfactory results.

These results clearly open relevant questions on the real, biological and clinical, significance of trying to combine different molecularly targeted agents for the treatment of cancer, either in a horizontal, or a vertical fashion. Besides 
the problems of toxicities and economical issues, once again the question is: is more better than less?

\section{References}

1. Wolpin BM, Mayer RJ (2008) Systemic treatment of colorectal cancer. Gastroenterology 134:1296-1310

2. Weihrauch MR, Stippel D, Fries JW et al (2008) Complete remission in a colon cancer patient with a large, irresectable liver metastasis after XELOX/cetuximab/bevacizumab treatment. Onkologie 31:464-467

3. Tol J, Koopman M, Cats A et al (2009) Chemotherapy, bevacizumab, and cetuximab in metastatic colorectal cancer. $\mathrm{N}$ Engl J Med 360:563-572

4. Linardou H, Dahabreh IJ, Kanaloupiti D et al (2008) Assessment of somatic k-RAS mutations as a mechanism associated with resistance to EGFR-targeted agents: a systematic review and metaanalysis of studies in advanced non-small-cell lung cancer and metastatic colorectal cancer. Lancet Oncol 9:962-972 